\title{
15
}

\section{Multimodal Longitudinal Journaling}

\author{
Lesley Jane Gourlay and Martin Oliver
}

\section{INTRODUCTION}

This chapter outlines the case for an holistic approach to studying e-learning, influenced by ethnography, New Literacy Studies (NLS) and sociomateriality (a tradition of work related to posthumanism and material semiotics). The theoretical framing for such work is presented and related to developments in ethnography and digital anthropology. To illustrate this approach, methods are described that were used to enact this framework in the context of a study of graduate students' digital literacies.

\section{METHODOLOGY IN E-LEARNING RESEARCH}

Research in e-learning is diverse. Czerniewicz (2010), in her analysis of research in the field over recent decades, argues that whilst a shared research agenda has started to emerge, the field remains inconsistent and fragmented. There is no homogenous perspective that shapes how research is undertaken, although some have tried to delineate the field in terms of the positivist, cognitivist approaches common within Instructional Design. However, the kinds of research published in educational technology journals remains diverse, most frequently adhering to the principles of social science, in that it is 'segmentally divided, interacting internally in complex ways' (p. 531). As a consequence, debates about methodology remain an important way of developing coherence and advancing the field: 
It is desirable for a horizontally segmented field to have a 'strong grammar'. In effect, this is what the call for more explicit and robust research is about: methods, theories and approaches that are robust, that are clearly articulated and that are carried through systematically. (Czerniewicz, 2010: 531)

Such debates are important for a second reason, too. Friesen (2009) also identifies a strong positivist, instrumental influence from Instructional Design, influenced by the concerns of the US military; this tradition, he argues, is preoccupied with developing a 'technical fix' for the problems of education. Such work has focused strongly on questions of tool design and on matters of practical implementation. As Friesen points out, such instrumentalism is celebrated, rather than challenged, by many researchers in the field (e.g. Koper, 2008: 356; Reeves, 2005: 304). Even where methodological development has taken place - for example, in relation to the emergence of design-based research (Barab \& Squire, 2004) - the primary contribution has, arguably, been to the improvement of designs so that they can support student engagement and learning outcomes more effectively (e.g. Dede et al., 2004), remaining focused on questions of efficiency rather than meaning or purpose.

This kind of instrumentalism accords well with the demands of policy makers, who are interested in questions of 'what works', in a way that masks more critical questions about the value of such practices, about alternatives (Oliver \& Conole, 2003) or about the possibility of developing theories that might explain these observations, by explaining why it works (Bennett \& Oliver, 2011). As has been argued, fashion and markets rather than principles or theory often appear to be the major driving force for e-learning research (Selwyn, 2007), and such uncritical, validating studies can bolster such orientations rather than challenging them.

Friesen argues, however, that there are alternatives. Drawing on Habermas (Freisen, 2009), he distinguishes instrumental approaches to research (focusing on technical interests) from those that are practically oriented (interested in interpretation or meaning) or 
emancipatory (concerned with critiques of power and control). He goes on to argue for the importance of developing and working with research methods that address these wider interests.

Instrumental approaches are commonly characterised by their focus on gains in effectiveness, efficiency or accessibility, framed in terms of pre-defined, often quantified metrics. Surveys relying on closed questions and those based on experiments would both be examples of approaches that support technical questions. Consequently, one way in which Friesen pursues his project is by drawing on alternative approaches that are influenced by phenomenographic traditions or which emphasise close, qualitative analysis. However, such alternative approaches are not necessarily easy to pursue. Part of the value attributed to elearning is that it supports study in a range of times and locations (Harasim, 2000); this can make work involving observations, ethnographies and other traditions that place an emphasis on close qualitative analysis problematic. Indeed, it could be argued that e-learning is most widespread precisely where ethnography is most challenging: where learners are distributed and remote (Oliver, 2000).

Some methods development has taken place to address these challenges, however - for example, within the context of the British JISC-funded programme of work exploring learners' experiences of e-learning (Sharpe et al., 2009). Whilst many of the projects Sharpe et al. discuss continued to rely on self-report satisfaction surveys, these came to be complemented by a growing range of alternative approaches, focusing on experiences, culture and context. These involved working with different forms of qualitative data, often in combination - such as the 'interview plus' approach, where discussions were grounded in relation to artefacts that learners had created, or to data describing their patterns of study, or 
to Interpretative Phenomenological Analysis, wherein learners were invited to generate narratives that explain meanings and experience.

Elsewhere, Jones and Healing (2010) explored learners' practice using a cultural probe exercise, in which undergraduate students were sent SMS text messages across a 24-hour period, recording answers to a fixed set of prompt questions either as video or in a notebook. This generated data about what students were doing, involving which technologies, where, with whom and how they felt about it, revealing the ways in which study had become integrated in students' life and leisure.

Such approaches can all be seen as reaching towards a more holistic, inclusive account of learners and their experiences, echoing the kinds of methodological developments that have taken place in related social sciences (Matusov, 2007), as well as in technical fields such as human-computer interaction (Grudin, 1990). In moving beyond questions of performance under controlled conditions, such research has drawn increasingly on ideas of social practice and on ethnographic sensibilities, in spite of the difficulties posed by the dispersal of study practices.

Recent policy around e-learning, such as the promotion of 'digital literacies' as a priority area for development, has made it increasingly important to develop such critical approaches. The increased dominance of 'skills' frameworks in funded research and development in elearning has arguably led to a focus on allegedly generic practices which may come to be regarded as free floating as opposed to socially situated. This type of focus may lead to a neoliberal discourse that frames digital practice as a bundle of 'employability skills' which are unproblematically acquired, homogenous, cumulative and endlessly transferable. Even when the complexities of the digital and the social are recognised, this perspective arguably continues to regard these capabilities as residing in the individual and amenable to training 
and development. These perspectives tend to seek as research outcomes, generalisability, 'impact' and cascading responses in terms of educational practice. If research is used at all, larger-scale methodologies are likely to be favoured, such as questionnaire research.

In contrast, a critique of such a position on e-learning and student practice might seek to investigate the particular, the emergent, the material and the situated - elements of the complexity of this phenomenon that closed, instrumental methods struggle to capture. This demands a methodology which would allow us to open up and investigate these subtle and difficult-to-access areas of educational day-to-day practice. Such approaches have been employed successfully in other practice-oriented areas, as evidenced for example by New Literacy Studies (NLS).

\section{Practice}

Focusing on textual practices, New Literacy Studies has provided a powerful critique of an area previously dominated by cognitivist perspectives which elided the importance of the social and the collective in educational research. It has also provided insights into the interplay between textual practice and subjectivities (Barton \& Hamilton, 2012). However, one limitation of this perspective is that in seeking to move away from the cognitive and towards the social, it has tended to place less emphasis on the embodied materiality of textual engagement, in terms of what students actually do, where they do it and what resources and artefacts they work with. (In this sense, 'materiality' refers to the physical practices involved in engagements with texts and digital technologies.) Through its emphasis on individuals and the texts themselves, some micro-level focus on the objects, devices and processes involved in textual engagement/production may have been de-emphasised, or naturalised as 'context'. Although the notion of context has been contested, and its complexity and dynamic nature have been recognised (e.g. Kell, 2003), the materiality of context remains relatively 
unexamined at a fine-grained level. A focus on the 'Digital Literacies' arguably necessitates a further interrogation of the notion of 'context' and the material, which can no longer be taken for granted. Although recent work has begun to integrate a greater focus on social semiotics in New Literacy Studies (e.g. Pahl and Rowsell, 2012), again the fine-grained materiality of literacy practices remains under-theorised. We argue that there is therefore a need for a framing which can adequately theorise textual engagement as not only socially situated and constitutive of subjectivities, but crucially also as digitally mediated sociomaterial practice.

What has become apparent in the intersection of this ethnographic approach to textual practice and recent work on digital literacies, is the importance of focusing on and understanding the day to day: looking at practices, not only outcomes or 'skills'. In educational research more broadly, the importance of classroom-based observational research and ethnographic methodologies to explore the detail of student engagement has become increasingly recognised. However, arguably, e-learning research has been slower to recognise that all engagement in educational process takes place in embodied and socially situated material contexts, including 'the digital'. A strong binary has conventionally been assumed in such research between 'distance' and 'face to face', as evidenced by the ongoing 'media effects' meta-studies that characterise these as binaries and seek to establish which is 'best' (see e.g. Reeves, 2005), but the complexities of contemporary social and educational practice reveal a situation in which this apparent boundary is increasingly blurred, breached and permeable.

\section{Materiality}

As has been argued, mainstream research into e-learning has tended to position the research participant as a 'user' of technologies, which are seen either explicitly or implicitly as 'tools'. This apparently commonsense framing is in fact highly ideological, in that it posits and 
underscores a clear division between the human and machine, action and context or writer/reader and digital text. Alternative framings of how agency is understood in engagement with technologies would question such strong binaries, instead proposing a more complex conception of social process which views online engagement and e-learning as a bricolage of artefacts and experiences.

This binary-based standpoint has been critiqued in the various strands of posthuman theory (e.g. Badmington, 2000; Wolfe, 2010), a theoretical stance which has questioned the dominant assumptions of humanism and the related assumptions on which much of social science and educational research methodology and analysis are based. The work of Donna Haraway (e.g. 1990) has been particularly influential in challenging apparently unassailable boundaries between categories of human and nonhuman such as human and animal, organism and machine, physical and non-physical. This work has been developed and related to technologies by Hayles, who has proposed the posthuman as a framing with which to interrogate contemporary social practice, arguing that 'the defining characteristics of the posthuman involve the construction of subjectivity, not the presence of nonbiological components' (1999: 4).

Actor-network theory (ANT) (e.g. Callon, 1986; Latour, 2005; Law \& Hassard, 1999) has also sought to challenge these categories, in particular emphasising the agency and centrality of nonhuman actors such as artefacts in social practice, as noted above. This has particular relevance for the study of digital education and e-learning where the human and machine interact, and has recently begun to be applied to educational contexts through study of the sociomaterial (e.g. Fenwick \& Edwards, 2010; Tummons, 2010).

For example, research evidence shows us that engagement with the digital in society and in education is not necessarily a smooth, disembodied, agentive transition from 'novice' to 
'expert' (e.g. Leander \& Lovorn, 2006; Fenwick et al., 2011). Instead, it may be seen as a highly complex and messy set of practices which are constantly shifting and emerging, intertwined with unstable settings which cannot be viewed as unchanging 'contexts' to human practice, but instead agentive forces (cf. Fenwick et al., 2011). These include multiple devices, information, software and wifi networks which are in constant flux. Additionally, the needs and priorities of the 'user' are equally complex, multilayered and volatile, combining the social, the work-related and the academic. Understanding this requires a close focus on the details of how everyday life is achieved.

In keeping with the principles of journaling as a methodology, ANT avoids the use of a priori categories (such as social class and gender), which tend to form the main analytical categories of mainstream sociology (Latour, 2005). Instead, the focus is on social practices enacted moment to moment in detailed, networked practices, drawing on ethnomethodology (e.g. Garfinkel, 1967) with its focus on the everyday and the 'micro'. The journaling methodology explored in this chapter also assumes that the small-scale, emergent actions of everyday material and embodied practice should be the focus of analysis, and in this respect represent a high degree of coherence with sociomaterial frameworks and perspectives. As Fenwick and Edwards argue, 'ANT's key contribution is to suggest analytic methods that honour the mess, disorder and ambivalences that order phenomena, including education' (2010: 1).

This chapter will provide a worked example of this theoretical and methodological stance within e-learning research, outlining a position that focuses on the materiality of lived experience. Drawing on theoretical work that emphasises embodiment and the situated materiality of practice (Latour, 2005; Fenwick et al., 2011), and which reveals the materiality of virtual resources (Hayles, 1999), we will argue that if we are to understand people's 
experiences of e-learning, it is important to ground studies in the day-to-day worlds of participants. As Fenwick et al. put it:

Humans, and what they take to be their learning and social process, do not float, distinct, in container-like contexts of education, such as classrooms or community sites, that can be conceptualised and dismissed as simply a wash of material stuff and spaces. The things that assemble these contexts, and incidentally the actions and bodies including human ones that are part of these assemblages, are continuously acting upon each other to bring forth and distribute, as well as to obscure and deny, knowledge. (Fenwick et al., 2011: vii)

\section{METHODOLOGICAL DEVELOPMENTS}

Given the general call for close analysis of qualitative data, and the importance of attending to the materiality of day-to-day practices, a strand of work has emerged in e-learning research that explores engagement from a fine-grained ethnographic perspective. This work explores the detail of practice as opposed to relying on self-account data in the form of questionnaires or interviews, and as such has done much to shed light on the complexities of engagement in online education. The development of methodologies loosely grouped as 'online ethnography', for example, has served to highlight the important differences and features of digital engagement that may have been overlooked in more mainstream approaches to social and educational research.

Researchers pursuing ethnographic enquiries have already engaged with many of the challenges associated with studying the development of digital culture. Some pursue this using fairly conventional ethnographic approaches, as with Malaby's study (2009) of the development of Second Life, in which the focus was on the culture of Linden Labs, the developers. However, others have found it necessary to develop new approaches in order to explore the way in which culture is played out online, as well as face to face. Hine (2000), for example, developed an approach that she called virtual ethnography, which combined 
participation in online communities with face-to-face site visits and interviews. This approach was able to provide valuable insights into the interplay between online and offline aspects of individuals' experiences, including the ways in which individuals' different forms of social practice overlapped, came into conflict, were managed and negotiated.

It has been argued that the emphasis on 'the digital' as an arena of practice can lead inadvertently to a binary focus, where 'the digital' is characterised as a rarified and separate realm fundamentally disconnected to the materiality of practice. Even studies such as Hine's, which address the inter-relationship of practices, can be seen as supporting the idea of parallel fields of cultural practice. The fact that all digital engagement takes place in embodied and socially situated settings can get lost in an analysis that focuses entirely on what can be observed or analysed online. Arguably, if the focus of research is on achieving a more complete and nuanced understanding of practice, then these methodologies may be combined to good effect with approaches that also investigate the material aspects of engagements with the digital.

It is worth speculating here on the influence of neoliberal discourses, which in this context have portrayed digital engagement as generic and 'free floating', and in doing so posit the human subject as a neutral, disembodied agent, unconnected to spatial, temporal and sociopolitical contexts (see e.g. Payton, 2012). This mainstream conception of digital devices and resources frames 'tools' as being something that can be appropriated unproblematically by the neoliberal subject. Arguably, this is a fantasy of ultimate efficiency and power, with technology in the service of unfettered human agency. This viewpoint supports the related 'skills' paradigm, which regards the ability to 'harness' technologies as a technical capability, which once mastered can be unproblematically transferred between settings. Undeniably, new mobilities have allowed engagement with the digital to take place in a broader range of 
spatial and temporal contexts than was previously the case. However, there is a risk in this analysis of collapsing into decontextualised and ideologically driven 'brave new world' discourses in society as a whole and in education in particular, which fail to take into account the micro-politics and complexities of embodied practice. The details of everyday device use, engagement with online texts and resources, uses of social media, the complex and intertwined relationship between digital and print literacy practices and artefacts - these cannot be fully accounted for by approaches which elide the materiality of practice.

Approaches that do address these concerns have been developed in the field of digital anthropology. Miller and Horst, for example, have laid out the challenges facing cultural studies concerned with digital practices (2012). Such work includes a strong commitment to holism, arguing 'that no one lives an entirely digital life and that no digital media or technologies exist outside of networks that include analogue and other media technologies' (p. 16), even if the analytical focus for any specific study is on digital practices. Moreover, they argue, materiality is the 'bedrock' for digital anthropology, in that digital infrastructure and technology are constituted materially; that the binary encoding of the digital is stored and operated on at the material level; and that the spaces, places and times in which digital practices are enacted shape these practices in important ways.

Similarly, actor-network theorists have argued that social practice cannot be understood purely in terms of human agency but should also consider 'non-human actors' - artefacts and entities which are integral to social practice and are seen as agentive within it (Latour, 2005). If this alternative, sociomaterial conception of digital practice is accepted, then research methodologies must be developed in order to investigate these networks of practice in all their complexity. As argued above, mainstream qualitative methodologies have been found to be limited, in that they may generate abstract responses that remain separate from the 
granularity of day-to-day practice and engagement, eliding the complexities of material practice and serving to underscore a 'digital dualism' (Jurgenson, 2011) that imagines the digital as a disembodied realm of unfettered agency.

Interviews and focus groups are an established and effective means of exploring participant motivations, experiences and viewpoints. Arguably, what is additionally required are methodologies which anchor these insights into the digital within the flow of day-to-day material practice. Participant observation is an established means to providing deep insights into day-to-day life. However, it is highly time-consuming, resource-intensive and, as noted earlier, it invokes particular practical and ethical challenges in the context of digital engagement, where a high proportion of participant behaviour is not amenable to observation in the public or semi-public domain.

Another criticism of interviews and focus groups is that they provide a 'snapshot' of experiences. In order to provide a more in-depth account, and particularly one able to provide insights into trajectories of personal development, it is useful to complement such approaches with longitudinal methods.

Journaling has also been used to document and explore the experiences of social actors, provides access to practice from the participant's point of view and enables a longitudinal perspective to be developed. Conventionally, this has tended to take the form of text-based entries, which confer the advantage of being relatively easy to analyse and collate. However, these can be regarded as onerous by participants, and unless carefully structured, may also lead to abstractions as opposed to reflections on particular experiences. As a result, methodologies have developed which allow for a broader range of modalities to be used, such as visual and audio-based journals. 
Visual methodologies (see e.g. Rose, 2012) are of particular utility in the complex arena of digital practice, as they can allow for the deployment of images in a way that offers several advantages.

Requiring research participants to express their perspectives or to document their experiences via journaling that involves nonverbal media (such as visual imagery in the form of photos, videos or drawings) can make the journaling task more engaging and less onerous than the construction of a solely text-based account. The conventional text-based journal may be perceived as a chore, difficult to maintain, and may also lead to the adoption of generic features of written expression such as the conventions of the personal diary or alternatively the formal and stylistic features of an academic text. Both of these genres may lead the participant to generate accounts which may become abstracted from the ethnographic context of practice at too early a stage in the data collection process, sacrificing granularity or particularity. In contrast, an image may confer the advantage of being a representation of a single instance, unlike a verbal token which stands for a category of experience. In this regard, the use of images may offer the feature of being rooted more specifically in the single instance or moment of social practice. An image may also serve as a powerful aide memoire in a way that a verbal account may not. Additionally, the visual presentation of an instance of social practice often includes the depiction of material artefacts and settings, which can become elided and somewhat 'lost' in more conventional forms of account data - where even in the case of vignettes and narrative analysis, the particular elements of the instance under discussion may become blurred and abstracted through the lenses of storytelling conventions, or indeed interview-based identity work and self-representation.

The immediacy and specificity of an image may also serve to enhance analysis at the stage of the interviews, where it may act as a powerful heuristic for discussion which can be linked 
back to the particular instance, and may in addition open up discussion of related instances. In this way, the image becomes not only a literal 'snapshot' record, but also a symbolic representation of a broader category of experience or practice, and may also function as a metaphor for difficult-to-access aspects of experience. In this way, the focus on an image may serve to circumvent the tendency of interviewees to focus on abstractions or generalisations in qualitative interviews, which can be a particular problem when asking participants to reflect on difficult-to-access, subtle or overlooked areas of social practice.

A further potential advantage of this approach is that, because the participants first create, then curate and then present the stimulus for the interviews, it can allow for a more agentive role for the research participants. This is not necessarily unique to work with images or visual data, but reflects the way in which this particular process relies on the images created and chosen by students who go on to shape the themes around which an interview may be organised - these themes emerging from the priorities expressed by the participants via their choices of image. This may be developed by asking participants to group images into categories and also to offer their own headings or analyses of the themes they see emerging in their library of images. Contemporary handheld devices with digital cameras and photo-based apps render these approaches increasingly accessible, although of course it should be borne in mind in some social settings that participants may not have access to (or familiarity with) these technologies. However, it might be argued that the rise in popularity of 'selfies' and the increased use of photo sharing in social networking may render visual methodologies more and more mainstream, as visual images are increasingly used to document and share personal experience and convey attitudes and identities online.

\section{LONGITUDINAL MULTIMODAL JOURNALING AND STUDENT DIGITAL ENGAGEMENT}


In order to illustrate the kinds of issues discussed above, this section draws on multimodal, longitudinal journaling data collected as part of a study of students' engagement with the digital in a higher education context.

\section{Framing the study}

'Digital literacies' are widely characterised as skills or competences (e.g. Martin \& Grudziecki, 2006), and this study was funded as part of a programme of work that had a strongly instrumental orientation, focused on 'those capabilities which fit an individual for living, learning and working in a digital society', with a strong emphasis on generic notions of employability (Payton, 2012). This framing has been roundly criticised for lacking any critical orientation and any disciplinary connection (Hinrichsen \& Coombs, 2014). More specifically, such 'skills' and 'capabilities' framings ignore the insights of New Literacy Studies, where it has been argued that literacies should be understood as situated, social practices of meaning making, rather than as decontextualised, stable attributes of individuals (Lea \& Street, 1998). Consequently, studies of literacies need to focus on textual practice (where 'text' is understood to be multimodal) and on the emergent struggles with meaning making that take place within such practices. Moreover, in the context of contemporary higher education, practice is saturated with digital mediation, even in the 'face-to-face' setting of the material campus (Gourlay, 2012). Consequently, the dominant framing of 'digital literacies' in this programme risks becoming both a checklist of technical 'skills' and a fantasy wishlist of the desirable attributes of the graduate's personal attributes (Gourlay et al., 2014). In order to move beyond this, this specific project drew on a definition of digital literacies developed by Gillen and Barton (2010: 9): 'the constantly changing practices through which people make traceable meanings using digital technologies'. This definition draws attention to the ongoing negotiation of practices and brings in the material semiotic perspectives developed in the context of actor-network theory (Gourlay et al., 2014). 


\section{Sampling frame}

In order to explore this, a study was undertaken at a large UK postgraduate institution specialising in educational research. The student body is predominantly mature and postgraduate, and many combine study with work and family responsibilities. Students are from diverse countries of origin and a broad range of educational cultures. Most have been out of formal education for several years. Consequently, they may never have used the kinds of digital technologies that are regarded as mainstream in higher education, although they have well-established repertoires of digital practices developed in personal or professional settings.

Focus groups were held to scope out themes and areas of practice in more detail. These focus groups involved four groups of students, reflecting the postgraduate population of the host institution: PGCE, taught masters, distance and doctoral. Participants were recruited to ensure a diversity of gender, age, home/EU or international, and full-time/part-time status. The discussions were transcribed and the thematic analysis of the transcripts was used to inform the next phase of work, which involved multimodal longitudinal journaling. Three students from each of the focus groups were invited to participate in the main study, providing a sample that ensured diversity against the criteria above, as shown in Table 15.1.

Table 15.1. Overview of the journaling participants

\begin{tabular}{|l|l|l|}
\hline Category & Pseudonym & Details \\
\hline MA & Nahid & M, 26, Bangladeshi \\
\hline MA & Juan & M, 30s, British \\
\hline MA & Yuki & F, 42, Japanese \\
\hline PhD & Django & F, 39, British \\
\hline PhD & Sally & F, 41, British \\
\hline PhD & Frederick & M, 25, German \\
\hline PGCE & Louise & F, 22, British \\
\hline
\end{tabular}




\begin{tabular}{|l|l|l|}
\hline PGCE & Faith & F, 30, Taiwanese \\
\hline PGCE & Polly & F, 40, British \\
\hline Distance & Bokeh & M, 30s, British \\
\hline Distance & Darren & M, 40s, American \\
\hline Distance & Lara & F, 40s, Chilean \\
\hline
\end{tabular}

\section{Data generation}

Participants were given iPod Touch devices and asked to document their day-to-day practices and interactions with texts and technologies in a range of settings. They were then asked to assemble and discuss these in a series of interviews. The study received institutional ethical clearance and followed approved procedures for informed consent, including guarantees of anonymity and confidentiality, and the right to opt out at any point.

The series of interviews involved 3-4 meetings over a period of 9-12 months. In the first of these, students were asked about their history of using technologies in education. As part of this, participants were asked to produce drawings of the places and resources that formed part of their studies; these formed the basis for a discussion of current practice and how this had developed. An example of such a map is shown in Error! Reference source not found. 


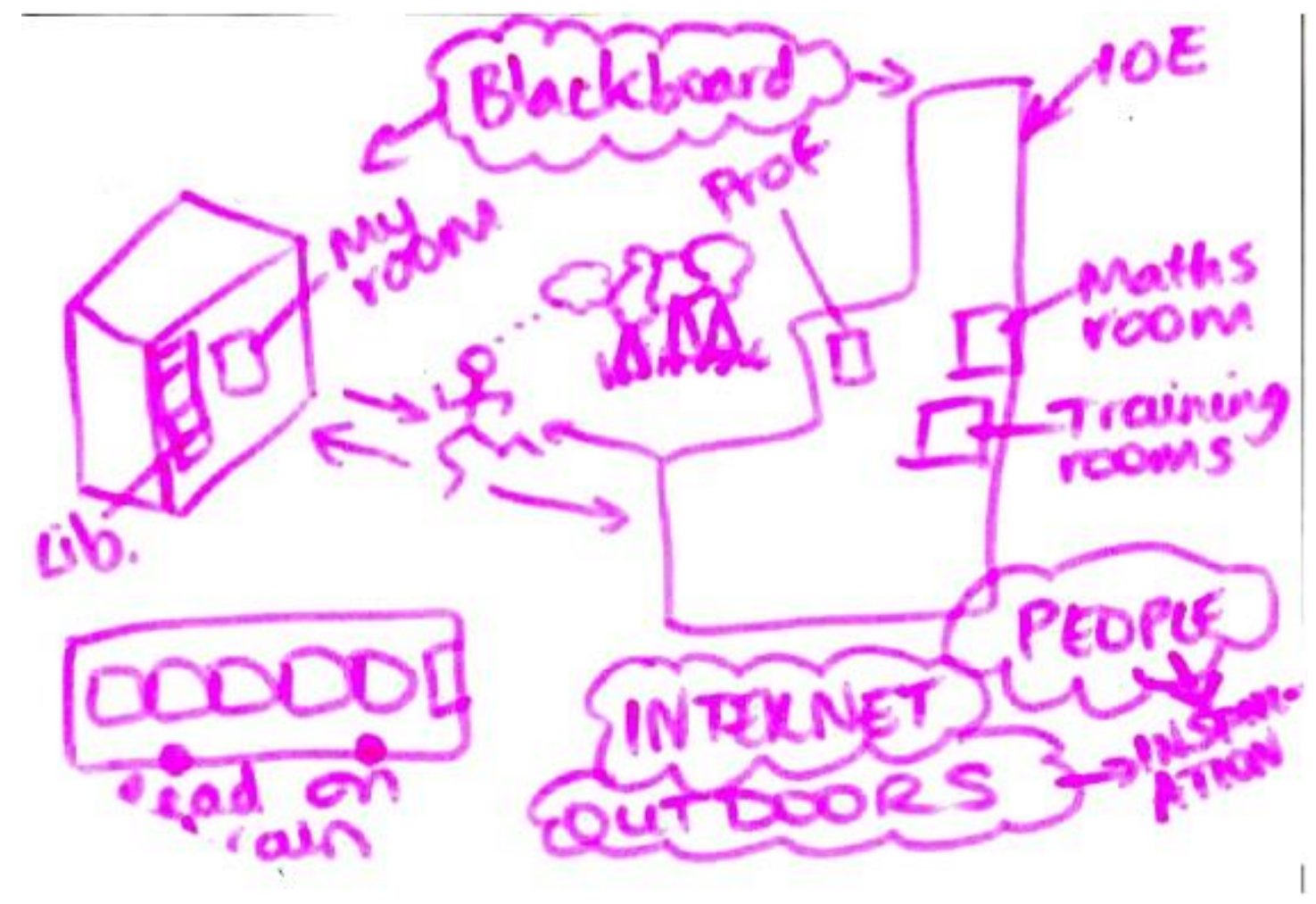

Figure 15.1. A student's map of their study practices

The discussion was followed by introducing the participants to the iPod Touch and explaining how they could use it to produce images, videos and textual notes. Participants were encouraged to focus on 'messy', micro-level, day-to-day lived activities. This focus was intended to reveal the networks that constituted practice, including material, spatial and temporal elements that ensured rootedness in everyday practices by drawing attention to objects and processes in a fine-grained way. These were elements that more conventional forms of data collection such as stand-alone interviews can obscure, since they rely on self-report and may lead to abstraction 
(Gourlay, 2010). Examples of images taken by students are shown in
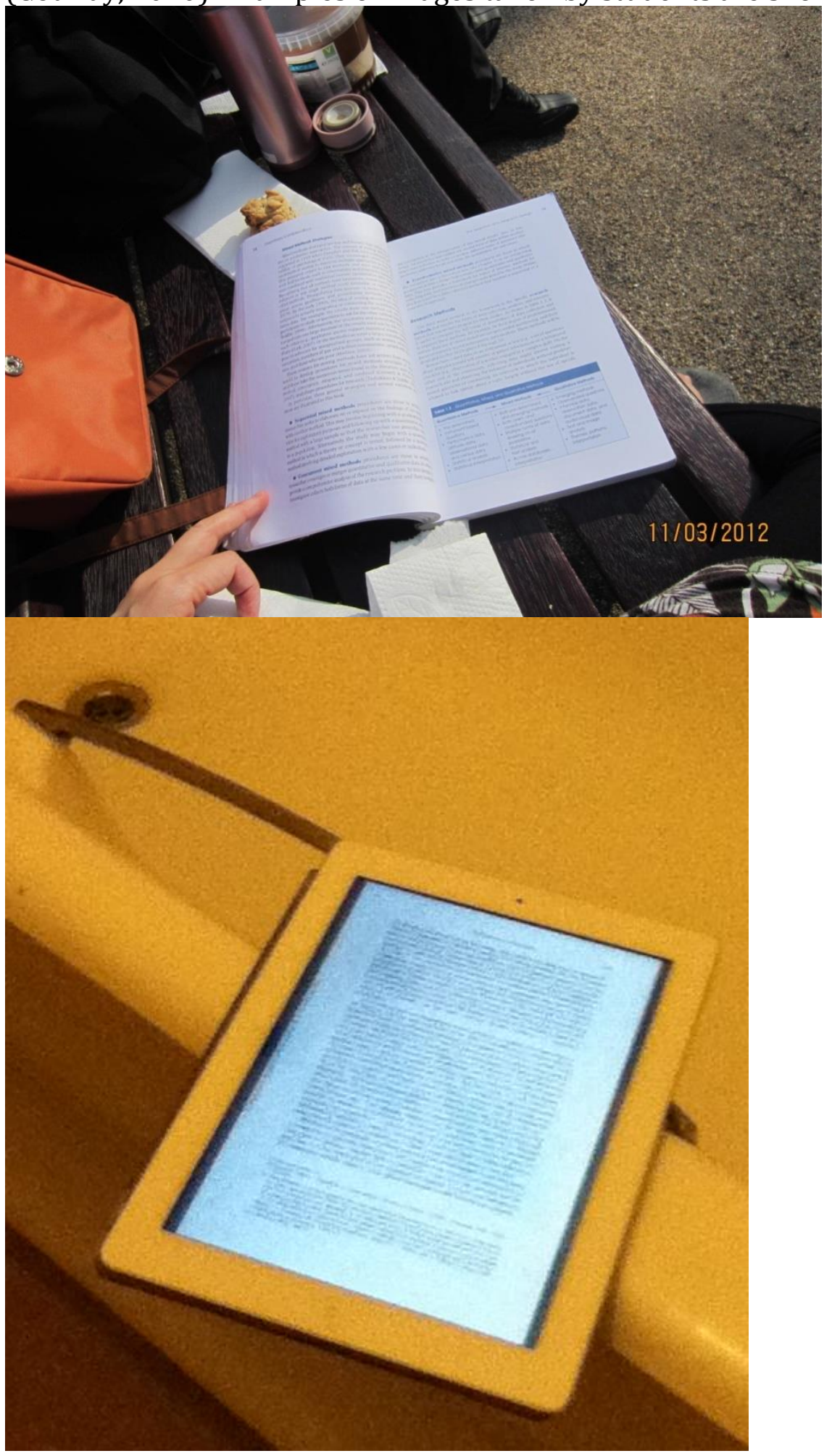

Figure 15.2. Images taken by students as part of their journaling work 

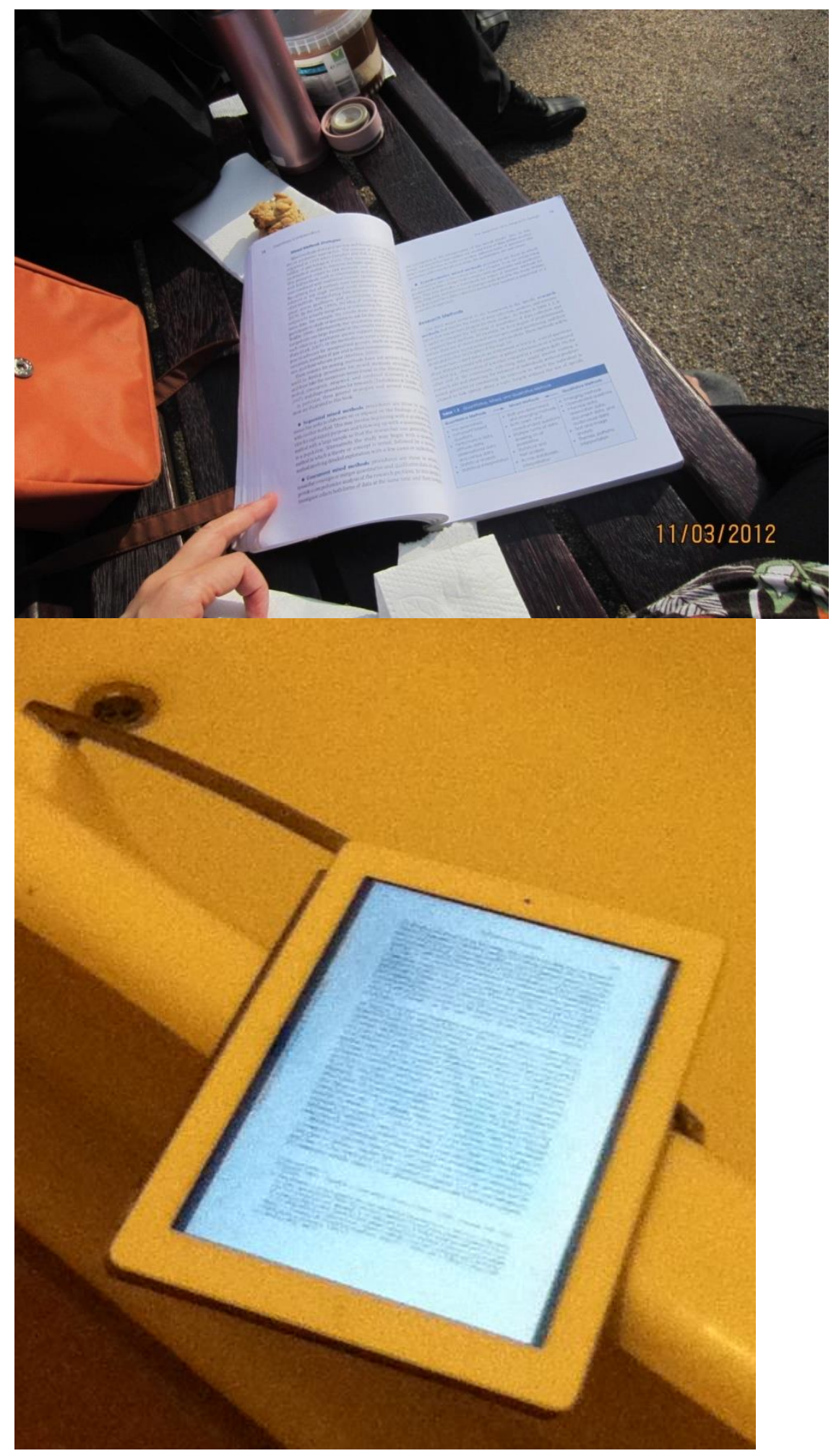

Figure 15.2. Images taken by students as part of their journaling work

Students were then given a month to undertake initial work, generating 'journals' of their practice constituted through a variety of multimodal data. They were then invited back for a second interview. In advance of this, they curated the data they had created, then in the 
discussion they reported on their experiences by presenting the journaling data to the interviewer and discussing them. These images and artefacts thus served not only as objects of analysis in their own right, but also as a stimulus for the in-depth exploration of subjectivities, challenges and issues - providing a rich set of participant accounts of technological engagement.

This process of participant fieldwork and interview was then repeated a further 2-3 times with each participant, narrowing the focus of the journaling and discussion each time. (The variation reflected both participants' willingness to participate and whether they felt they still had new points to make with their journals.) For these later interviews, the participants were encouraged to focus on institutional infrastructure (the library, the course Virtual Learning Environment or VLE) and textual practice (working with source material such as data sets or library texts, and the production of assessed work such as essays, chapters or dissertations).

As the interviews progressed, the participants were encouraged to take greater responsibility for the curation, presentation and interpretation of their multimodal journaling data. For example, some students chose to create presentations, using PowerPoint or Prezi, which combined images with text that either provided explanations or else associated the image with a theme, providing an interpretative frame (see Figure 15.3). 

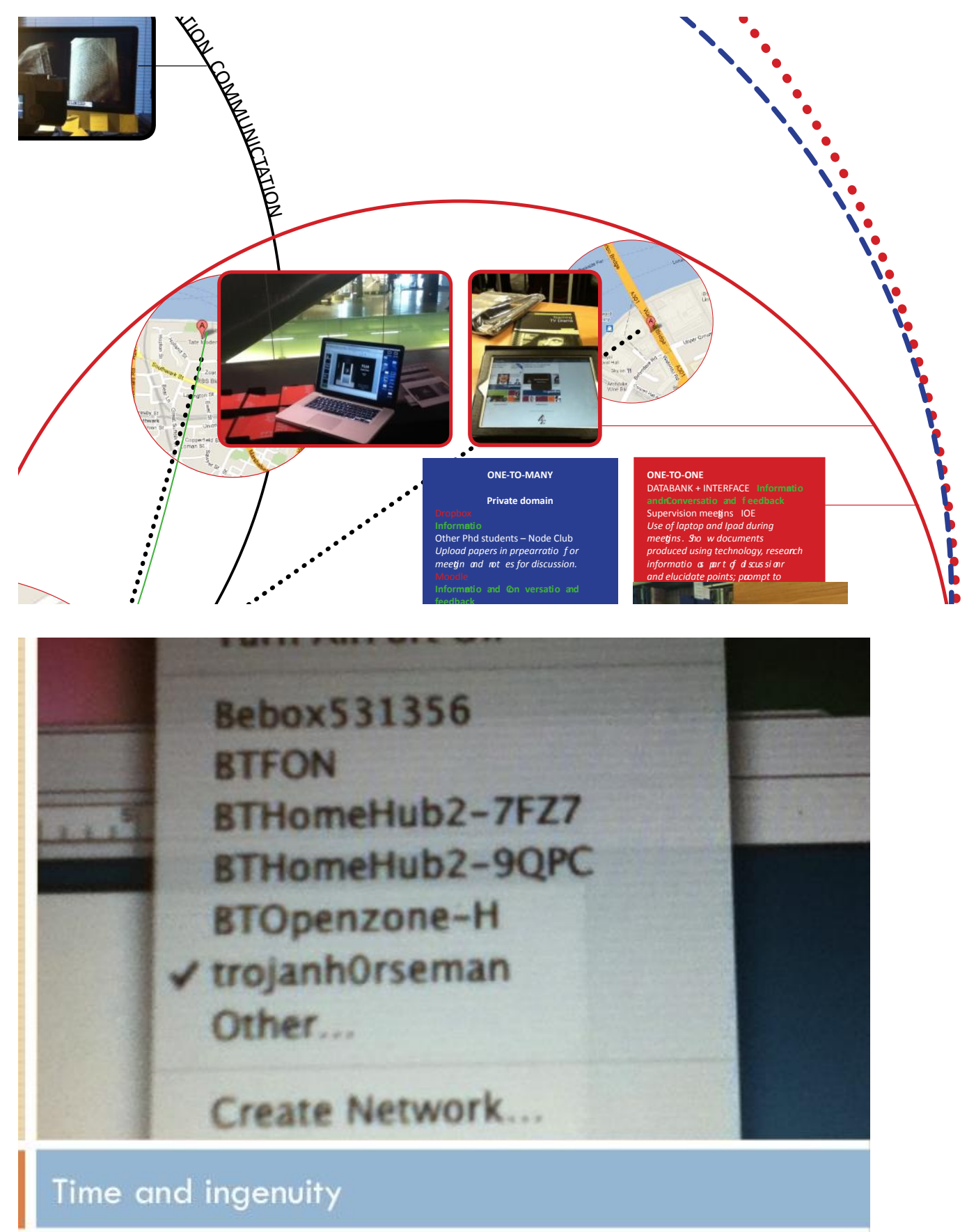

I have spent a massive amount of time trying to do fixes, patches and workarounds, due to obsolete hardware,
compatibility etc. This has however developed my knowledge and ingenuity. I would only dare do this on a Mac
though. In this picture I used a scary name to deter people from my unencrypted portable hotspot. I also use internet
forums a lot to look for fixes.

Figure 15.3. Slides from students' presentations of their journaling data

In addition, many participants brought in the material artefacts of their practices, presenting these alongside the digitised words and images that they had curated. These included books, notebooks, highlighter pens, post-it notes, folders, printed papers and completed surveys. 
This creation of digital and material assemblages by students was methodologically important. ANT research has been criticised for a lack for reflexivity, whereas NLS research has developed approaches in which participants contribute to the interpretation and analysis of their data. Encouraging participants to create, curate, frame and present their data in this way can therefore 'underpin a research agenda which combines the detachment and symmetry of ANT ethnography with a commitment to reflectivity and collaboration' (Clarke, 2002: 120).

\section{Data analysis}

A first descriptive pass through the data allowed several simple but important conclusions to be drawn. The data participants created made it clear, for example, that their experience of engaging with institutional infrastructures (such as course VLEs or library databases) varied both across and within groups. The structuring of course VLE sites provided a strong framing of the institution for distance students, but was minimal and hardly relevant for doctoral students. Use of library databases depended in part on whether participants were already familiar with professional research databases, such as PubMed for those from a medical background. This undermined the widespread discourse of 'the student experience', showing that participants' experiences were not singular, and that aggregation and 'averaging out' of their responses hid important differences (Gourlay \& Oliver, 2014).

Similarly, simply listing the technologies used by students undercut the credibility of taxonomic lists of skills that might be understood as constituting 'digital literacies'. The technologies were wide-ranging, including office tools, VLEs, email, databases, synchronous conferencing software, social networking sites, image-editing software, reference management software, data management and analysis software, GPS services, and devices including desktop PCs, laptops, tablet computers, mobile phones and e-book readers. For any 
given participant, only a subset of these was relevant: for example, distance students made frequent use of synchronous conferencing software such as Skype or Elluminate, whereas campus-based students did not; GPS software was only relevant for those undertaking fieldwork. Participants used a variety of different examples of these technologies, not all of which were interchangeable (such as Mendeley and Endnote). They also worked with multiple examples of some categories of technology, such as in using Facebook, Academia.edu and LinkedIn to manage the separation of personal, academic and professional networks. Their use also varied over time, for example because students had stopped using SPSS and could no longer remember how to make the software work for them, particularly as new versions had been released. Consequently, far from 'digital literacies' being stable and taxonomic, as assumed elsewhere in the research programme, the data set provided evidence that technology use varied by discipline, programme of study, kind of text being produced, social framing and time.

Moreover, the constant representation and presence of non-digital artefacts (such as the folders, books, pens and post-it notes that the participants brought to interviews) undermined the idea that 'digital literacies' form a neatly bounded area of practice. The physical presence of these material artefacts emphasised the continued importance of print literacies, and their narratives in the interviews showed the complex way in which digital and print literacies were interwoven and interdependent.

As noted above, the visual data generated by students served a double purpose: it acted to initiate and ground discussion in the interviews, but also formed a corpus of data in its own right. The maps drawn by students in the first interview provide a clear example of this. Juan, for example, drew a map in which study spaces were clustered together at the right-hand edge of his piece of paper, a floorplan of his flat was shown at the far left of the paper and the 
middle was left mainly as white space, except for a dashed line drawn from top to bottom right in the middle. This image can be read as showing the attempted separation of study practices from home life, with the space indicating an attempt to maximise this, and the gaps in the separating line, the permeability of these practice settings. These were themes that Juan also developed in the interview and to which he returned with photographs and in his presentations in subsequent interviews.

This kind of descriptive overview served to undermine common generalisations; however, it did not in itself provide alternative framings of digital literacy. A next step towards this involved a theoretical description of the data set. In this, the data were related to concepts central to the alternative framing of 'digital literacies'. For example, the sociomaterial reframing of digital literacies introduced the idea of nonhuman actors having a role in explaining the success (or otherwise) of successful digital literacy practices. There was evidence in the data set that corroborated this:

\section{My third half of my brain is Google scholar. (Frederick Interview 2)}

This kind of thematic analysis also helped to identify variations or challenges to this conceptual framing. For example, there was a considerable volume of data that emphasised the continued importance of non-digital literacies, the ongoing role of conventional texts as part of academic study:

My favourite way of studying something is sitting down with a book and ... a pen and some yellow paper and taking notes ... And then I will use the technological side as well, because ... Yes, I like combining the two, but I also like to be ... the demarcation lines between them, you know, if I, if I have a reading to do then I can, then I almost, I invariably print it off and highlight. (Juan Interview 1)

This mapping of supporting and challenging data led to a further phase of analysis, in which patterns of concepts were identified as being useful in delineating different patterns of 
practice. For example, the ways in which individuals described their relationship with agentive technologies formed an important part in delineating three orientations towards digital literacy practices: curation, combat and coping. (These orientations were not attached to individuals as some kind of stable characteristic, since all individuals provided examples of more than one orientation; instead, they were used to characterise patterns of practice.)

The 'curation' orientation characterised patterns of behaviour in which multimodal resources were created, brought together, managed and used in the production of new texts. Yuki, for example, recorded lectures, digitised texts and accessed online resources, using her iPad as a definitive place where all these resources were organised and through which they could be accessed. She was therefore able to engage in complex, inter-textual reading and practices:

For example when I attend a lecture or a session I always record the session ... Sometimes I listen to the lecture again to confirm my knowledge or reflect the session ... For example we're writing an essay and I have to confirm what the lecturer said, I could confirm with the recording data. (Yuki Interview 1)

The 'combat' orientation also involved working with a diverse array of multimodal texts, but discussion of these practices was characterised by a sense of threat and struggle. While the 'curation' orientation emphasised the agency of the participant, the 'combat' orientation emphasised the agency of the devices and services with which the participant was required to engage:

I feel like, also that Google is equally watching you. You know, they're all watching you, they're all trying to sell you things, and the thing is not, I don't so much mind being bombarded with advertising as I mind having things put about me on things like Facebook that I don't want. You know, I don't want my friends to spy on me, I don't want my friends to know what I listen to on YouTube. (Sally Interview 1) 
The 'coping' orientation was marked by failures, either experienced or threatened. In both the previous orientations, the participant was presented as having a sense of agency (even if other actants were able to 'push back' against this); in the 'coping' orientation, participants were characterised by their lack of agency and by the consequent struggle to achieve things:

I think they (the technologies) control me as well, because I can't really do anything without them. (Faith Interview 1)

I don't feel I'm kind of like I'm really up to date with computers compared to a lot of people, but I feel like you just have to keep moving otherwise you will get left behind. (Sally Interview 1)

Identifying orientations such as these has implications for educational strategies and practices. In contemporary higher education, there is, for example, widespread discussion of 'the student experience', often linked to conceptions of students as consumers, and which has been positioned as a foundational reference point for sector-wide policy (see e.g. Ramsden, 2008). Such discussions frequently rest upon responses to national or institutional responses to student satisfaction surveys, linking developments to dominant or average ('normal') responses. Orientations such as those outlined here undermine such unified accounts: they show that experiences can be inconsistent, even incompatible. They are also situated responses, not 'types' of students; resisting the temptation to attach these to supposedly freefloating individuals allows for the possibility that people might change, or be inconsistent; they might orient one way today, and another tomorrow, as their circumstances change. This demands a more nuanced and localised kind of response, one that attends to the particularities of these students, at this moment, rather than offering a 'one size fits all' solution.

The final section will summarise the ways in which longitudinal multimodal journaling, as used in this project, uncovered aspects of student experience and practice that would have been less apparent or occluded in more conventional approaches, as argued for in the earlier 
sections of this chapter. It will also highlight some of the challenges this methodology presents in terms of practical data collection and analysis.

\section{DISCUSSION}

The analysis above has been supplied in order to exemplify the types of data about e-learning which can be produced using the methodological approach of longitudinal multimodal journaling. As illustrated above, the approach led to a deepening of focus and analysis over time in collaboration with participants. Throughout the project, there was a sustained and consistent focus on the fine-grained and the particular. Artefacts and materiality in the form of texts, devices and a range of objects were represented and discussed throughout the data collection and analytic process, with analytical categories only reached gradually and collaboratively. The analysis resisted any tendency to generalise strongly beyond the context, although it should be noted that the findings are consistent with several closely related studies (Asher \& Duke, 2012) and so could be regarded as generating implications for future research and practice.

However, it should also be recognised that such an approach presents challenges in addition to conferring advantages. In terms of research ethics, particular care must be taken around the use of images and individual privacy. Additionally, there is clearly a tension between depth and breadth in this form of longer-term research, as the sustained period of detailed data generation tends to make projects of this type with large numbers of participants prohibitively resource-intensive and challenging to manage. Considerable researcher and participant time is required, and incentives are necessary in order to sustain participation. Saturation and/or repetition is a further risk with repeated interviews, and care must be taken to provide appropriate guidance and variety around journaling to avoid this effect and to ensure progression and depth. However, researchers must also reflect on the extent to which 
multimodal journaling should be structured and guided, as opposed to spontaneous and freeform - a balance needs to be struck. Additionally, although recent versions of text-tagging software such as NVivo can now embed non-textual media, greatly aiding the analysis process and the integration of multimodal data, tensions still arise between the generation of a large and rich data set and the manageability of data collection and analysis. A further question arises as to the extent to which the visual images should be regarded simply as aide memoires, illustrations or objects of analysis in their own right (Gourlay, 2010). A final complication lies in the ongoing resistance of mainstream academic journals in social science and education to recognise visual images as data, often leading to a reluctance to reproduce more than one or two images in a publication, exacerbated by limits on space.

Overall, such a research approach requires careful design, flexibility and adequate resources in order to do justice to the complexity of the data generated. However, despite these caveats we would argue that the use of multimodal journaling offers access to a range

of fine-grained, embodied, situated insights into the lived experience of what we call 'elearning' in unexpected and fresh formats not generally accessed by mainstream research methodologies.

\section{REFERENCES}

Asher, A. \& Duke., L. (2012) College Libraries and Student Culture: What We Now Know. Chicago: American Library Association.

Badmington, N. (2000) Posthumanism. Basingstoke: Palgrave.

Barab, S. \& Squire, K. (2004) Design-based research: putting a stake in the ground. The Journal of the Learning Sciences, 13(1), 1-14. 
Barton, D. \& Hamilton, M. (2012) Local Literacies: A Study of Reading and Writing in One Community. London: Routledge.

Bennett, S. \& Oliver, M. (2011) Talking back to theory: the missed opportunities in learning technology research. Research in Learning Technology, 19(3), 179-189.

Callon, M. (1986) Some elements of a sociology of translation: domestication of the scallops and the fishermen of St Brieuc Bay. In Law, J. (ed.), Power, Action and Belief: A New Sociology of Knowledge? London: Routledge \& Kegan Paul.

Clarke, J. (2002) A new kind of symmetry: actor-network theories and the new literacy studies. Studies in the Education of Adults, 34(2), 107-122.

Czerniewicz, L. (2010). Educational technology: mapping the terrain with Bernstein as cartographer. Journal of Computer Assisted Learning, 26(6), 523-534.

Dede, C., Nelson, B., Jass Ketelhut, D., Clarke, J. \& Bowman, C. (2004). Design-based research strategies for studying situated learning in a multi-user virtual environment. Paper presented at the 6th International Conference on Learning Sciences, Santa Monica, CA.

Fenwick, T. \& Edwards, R. (2010) Actor-Network Theory in Education. London: Routledge. Fenwick, T., Edwards, R. \& Sawchuk, P. (2011) Emerging Approaches to Educational Research: Tracing the Sociomaterial. London: Routledge.

Friesen, N. (2009) Rethinking E-learning Research. New York: Peter Lang.

Garfinkel, H. (1967) Studies in Ethnomethodology. Englewood Cliffs, NJ: Prentice-Hall. 
Gillen, J. \& Barton, D. (2010) Digital Literacies: A Research Briefing by the Technology Enhanced Learning Phase of the Teaching and Learning Research Programme. London: London Knowledge Lab. Online: www.tlrp.org/docs/DigitalLiteracies.pdf

Gourlay, L. (2010) Multimodality, visual methodologies and higher education. In SavinBaden, M. \& Howell Major, C. (eds), New Approaches to Qualitative Research: Wisdom and Uncertainty, pp. 80-88. London: Routledge.

Gourlay, L. (2012) Cyborg ontologies and the lecturer's voice: a posthuman reading of the 'face-to-face'. Learning, Media and Technology, 37(2), 198-211.

Gourlay, L. \& Oliver, M. (2014) Why it's not all about the learner: a sociomaterial account of students' digital literacy practices. Paper presented at Networked Learning 2014, Edinburgh. Online: www.networkedlearningconference.org.uk/abstracts/pdf/gourlay

Gourlay, L., Hamilton, M. \& Lea, M. (2014) Textual practices in the new media digital landscape: messing with digital literacies. Research in Learning Technology, 21: 21438. Online: www.researchinlearningtechnology.net/index.php/rlt/article/view/21438.

Grudin, J. (1990) The computer reaches out: the historical continuity of interface design. In Proceedings of the SIGCHI Conference on Human Factors in Computing Systems, pp. 261268. New York: ACM.

Harasim, L. (2000) Shift happens: online education as a new paradigm in learning. The Internet and Higher Education, 3(1), 41-61.

Haraway, D. (1990) A manifesto for cyborgs: science, technology, and socialist feminism in the 1980s. In Nicholson, L. (ed.), Feminism/Postmodernism, pp. 190-233. New York: Routledge. 
Hayles, N. K. (1999) How We Became Posthuman: Virtual Bodies in Cybernetics, Literature, and Informatics. Chicago: University of Chicago Press.

Hine, C. (2000). Virtual Ethnography. London: Sage.

Hinrichsen, J. \& Coombs, A. (2014) The five resources of critical digital literacy: a framework for curriculum integration. Research in Learning Technology, 21: 21334. Online: www.researchinlearningtechnology.net/index.php/rlt/article/view/21334

Jones, C. \& Healing, G (2010) Networks and locations for student learning. Learning, Media and Technology, 35(4), 369-385.

Jurgenson, N. (2011) Digital dualism versus augmented reality. Cybergology: The Society Pages, 24.

Kell, C. (2003) Everything has a place: the ins and outs of context. Fine Print: Journal of Adult English Language and Literacy Education, 26, 12-19.

Koper, R. (2008) Open source and open standards. In Spector, J., Merrill, M., van Merriënboer, J. \& Driscol, M. (eds), Handbook of Research on Educational Communications and Technology (3rd edn), pp. 355-368. New York: Routledge.

Latour, B. (2005) Reassembling the Social: An Introduction to Actor-Network Theory. Oxford: Oxford University Press.

Law, J. \& Hassard, J. (1999) Actor-Network Theory and After. Oxford: Blackwell.

Lea, M. \& Street, B. (1998) Student writing in higher education: an academic literacies approach. Studies in Higher Education, 23(2), 157-172. 
Leander, K. \& Lovorn, J. (2006) Literacy networks: following the circulation of texts, bodies and texts in the schooling and online gaming of one youth. Cognition and Instruction, 24(3), 291-340.

Malaby, T. (2009) Making Virtual Worlds: Linden Lab and Second Life. London: Cornell University Press.

Martin, A. \& Grudziecki, J. (2006). DigEuLit: concepts and tools for digital literacy development. Innovation in Teaching and Learning in Information and Computer Sciences, 5(4), 249-267.

Matusov, E. (2007) In search of the 'appropriate' unit of analysis for sociocultural research. Culture \& Psychology, 13(3), 307-333.

Miller, D. \& Horst, H. (2012) The digital and the human: a prospectus for digital anthropology. In Horst, H. \& Miller, D. (eds), Digital Anthropology, pp. 3-35. London: Berg. Oliver, M. (2000) Evaluating online teaching and learning. Information Services \& Use, 20(2/3), 83-94.

Oliver, M. \& Conole, G. (2003) Evidence-based practice and e-learning in higher education: can we and should we? Research Papers in Education, 18(4), 385-397.

Pahl, K. \& Rowsell, J. (2012) Literacy and Education (2nd edn). London: Sage.

Payton, S. (2012) JISC Briefing: Developing Digital Literacies, Bristol. Online: www.jisc.ac.uk/media/documents/publications/briefingpaper/2012/Developing_Digital_Liter acies.pdf 
Ramsden, P. (2008) The Future of Higher Education Teaching and the Student Experience. York: The Higher Education Academy.

Reeves, T. (2005) No significant differences revisited: a historical perspective on the research informing contemporary online learning. In G. Kearsley (ed.), Online Learning: Personal Reflections on the Transformation of Education, pp. 299-308. Englewood Cliffs, NJ:

Educational Technology Publications.

Rose, G. (2012) Visual Methodologies: An Introduction to the Interpretation of Visual Methods. London: Sage.

Selwyn, N. (2007) The use of computer technology in university teaching and learning: a critical perspective. Journal of Computer Assisted Learning, 23(2), 83-94.

Sharpe, R., Beetham, H., Benfield, G., DeCicco, E. \& Lessner, E. (2009) Learners experiences of e-learning synthesis report: explaining learner differences. Online: http://jisc.ac.uk/media/documents/programmes/elearningpedagogy/lxp2finalsynthesis.pdf Tummons, J. (2010) Institutional ethnography and actor-network theory: a framework for researching the assessment of trainee teachers. Ethnography and Education, 5(3), 345-357.

Wolfe, C. (2010) What is Posthumanism? Minneapolis, MN: University of Minnesota Press. 